\title{
DESEMPENHO E CARACTERÍSTICAS DE CARCAÇAS DE FÊMEAS SUÍNAS ALIMENTADAS COM FARELO DE ALGODÃO
}

\author{
Cristina Lucia Michaello Macêdo do Nascimento ${ }^{1}$ \\ Wilson Moreira Dutra Junior ${ }^{2}$ \\ Carlos Boa Viagem Rabello² \\ Maria do Carmo Mohaupt Marques Ludke 2 \\ Guilherme Rodrigues Nascimento ${ }^{3}$ \\ Liliane Olímpio Palhares ${ }^{4}$
}

NASCIMENTO, C. L. M. M. do; DUTRA JUNIOR, W. M.; RABELLO, C. B. V.; LUDKE, M. do C. M. M.; NASCIMENTO, G. R.; PALHARES, L. O. Desempenho e características de carcaças de fêmeas suínas alimentadas com farelo de algodão. Arquivos de Ciências Veterinárias e Zoologia da UNIPAR, Umuarama, v. 23, n. 1cont., e2302, 2020.

RESUMO: Objetivou-se avaliar os parâmetros de desempenho, características de carcaças e viabilidade econômica de fêmeas suínas alimentadas com diferentes níveis de inclusão do farelo de algodão (FA) nas rações. Foram utilizadas 24 fêmeas suínas, com peso vivo médio de 48,20 $\pm 5,44 \mathrm{~kg}$ (fase de crescimento) e de 87,62 $\pm 6,72 \mathrm{~kg}$ (fase de terminação). O delineamento foi em blocos casualizados, com quatro tratamentos $(0 \%, 10 \%, 20 \%$ e 30\% de inclusão de farelo de algodão), seis repetições, um animal por unidade experimental. $\mathrm{Na}$ fase de crescimento os parâmetros de desempenho não foram influenciados significativamente, na fase de terminação e total houve uma redução do ganho de peso e consumo de ração. Para as características de carcaça os animais apresentaram redução na espessura de toucinho e um aumento na área de olho de lombo até o nível de 13,5\% de inclusão de FA. Quanto ao peso dos órgãos houve um aumento do peso do coração, fígado e rins. Conclui-se que é possível incluir o FA até o nível de 30\%, sem afetar as características de desempenho na fase de crescimento, e de até $13,5 \%$ sem comprometer o rendimento de carcaças.

PALAVRAS-CHAVE: Alimento protéico. Ganho de peso. Rendimento de carcaça. Suínos.

\section{PERFORMANCE AND CARCASS CHARACTERISTICS IN FEMALE PIGS FED WITH COTTONSEED MEAL}

\begin{abstract}
The purpose of this study was to evaluate the performance, carcass characteristics and economic viability parameters in female pigs fed with different levels of cottonseed meal (CM) in the diets. Twenty-four crossbred female pigs were used, with live weight of $48.20 \pm 5.44 \mathrm{~kg}$ (growth phase), and $87.62 \pm 6.72 \mathrm{~kg}$ (finish phase). A completely randomized block design with four treatments $(0 \%, 10 \%, 20 \%$ and $30 \%$ inclusion of cottonseed meal) was used with six replication, one animal per experimental unit. The performance parameters were not significant in the growth phase; the finish and total phase presented a reduction of weight gain and feed intake. For the carcass characteristics, the animals presented a reduction in backfat thickness, and an increase in the loin area up to $13.5 \% \mathrm{CM}$ inclusion level. The organs showed weight increase for the heart, liver, and kidneys. It can be concluded that CM can be included in up to 30\% without affecting the performance characteristics in the growth phase, and up to $13.5 \%$ without compromising the carcass characteristics.
\end{abstract}

KEYWORDS: Carcass yield. Protein food. Pigs. Weight gain.

\section{RENDIMIENTO Y CARACTERÍSTICAS DE CAPARAZÓN DE HEMBRAS PORCINAS ALIMENTADAS COM HARINA DE ALGODÓN}

RESUMEN: El objetivo de este estudio ha sido evaluar los parámetros de rendimiento, características de caparazón y la viabilidad económica de hembras porcinas alimentadas con diferentes niveles de inclusión de harina de algodón (HA) en las raciones. Se utilizaron veinticuatro hembras porcinas, con peso vivo promedio de 48,20 $\pm 5,44 \mathrm{~kg}$ (fase de crecimiento) y $87,62 \pm 6,72 \mathrm{~kg}$ (fase de terminación). El diseño fue en bloques aleatorios, con cuatro tratamientos $(0 \%, 10 \%, 20 \%$ y $30 \%$ de inclusión de harina de algodón), seis repeticiones, un animal por unidad experimental. En la fase de crecimiento, los parámetros de rendimiento no se vieron significativamente influenciados; en la fase de terminación y total, hubo una reducción en el aumento de peso y la ingesta de pienso. Para las características de caparazón, los animales mostraron una reducción en el espesor del tocino y un aumento en el área del ojo del lomo, hasta el nivel del 13,5\% de la inclusión de HA. En cuanto al peso de los órganos, hubo un aumento en el peso del corazón, hígado y riñones. Se concluye que es posible incluir HA hasta el nivel de $30 \%$, sin afectar las características de rendimiento en la fase de crecimiento, y de hasta un $13,5 \%$ sin comprometer el rendimiento de los caparazones.

PALABRAS CLAVE: Alimentos proteicos. Aumento de peso. Rendimiento de caparazón. Porcinos.

\footnotetext{
DOI: 10.25110 /arqvet.v23i1.2020.7194

${ }^{1}$ Alunos de pós-graduação - Universidade Federal Rural de Pernambuco - Recife, PE.

${ }^{2}$ Professor Adjunto/Titular - Universidade Federal Rural de Pernambuco - Recife, PE.

${ }^{3}$ Professor - Instituto Federal Baiano Campus Santa Inês - BA.

${ }^{4}$ Universidade Federal Rural de Pernambuco. lilianepalhares@zootecnista.com.br
} 


\section{Introdução}

Historicamente a cadeia produtiva da suinocultura tem desenvolvido significativamente ao longo das últimas décadas tornando-se uma atividade rentável e competitiva (THORNTON, 2010). A viabilidade econômica na suinocultura depende do preço e da disponibilidade local dos grãos, pois o custo da ração pode representar até $70 \%$ do custo da produção, refletindo no preço pago por quilograma de carne suína. A utilização de alimentos alternativos aos convencionais, milho e farelo de soja, é interessante desde que atenda às exigências de nutrientes a um menor custo sem afetar negativamente o desempenho dos animais e, a eficiência de produção (BORUAH et al., 2018).

$\mathrm{O}$ farelo de algodão (FA) representa mundialmente a segunda mais importante fonte proteica disponível para a alimentação animal, ultrapassada apenas pela soja (BARROS et al., 2011). O FA apresenta teores entre 28,1 e 42,9\% de proteína bruta (PB) (ROSTAGNO et al., 2017), tornando-o um potencial substituto ao farelo de soja em rações para suínos nas diferentes fases de criação (MOREIRA et al., 2006). Entretanto, seu emprego na alimentação de não ruminantes como único suplemento proteico das dietas é limitado (PAIANO et al., 2006). Apesar de apresentar elevado nível de proteína, sua utilização para animais não ruminantes possui alguns obstáculos como o nível de fibra bruta.

Alimentos com elevado teor de fibra podem aumentar a peristaltismo, acelerando o trânsito intestinal, tanto no intestino delgado quanto no intestino grosso, ocasionando a redução do tempo de contato com as enzimas digestivas, bem como diminuindo o tempo disponível para absorção de nutrientes (NEPOMUCENO et al., 2016). As fibras podem ser um componente crítico em rações de animais não ruminantes, pois quando presente em grande quantidade podem limitar a produtividade animal (GOMES et al., 2007), comprometendo os parâmetros de desempenho e características de carcaça.

O farelo de algodão também possui princípio tóxico, o gossipol, um aldeído polifenólico produzido nas glândulas de secreção interna do algodoeiro que conferem à planta resistência contra pragas. É um pigmento amarelo tóxico que age como inibidor da atividade de diversas enzimas (BLEVINS et al., 2010). Segundo os mesmos autores, devese ter prudência ao fornecer esse ingrediente aos animais, pois a substância possui efeito tóxico aos animais, podendo comprometer as funções hepáticas, taxa respiratória e a capacidade de transporte do oxigênio pelos eritrócitos, possibilitando o ataque cardíaco, os efeitos tóxicos são cumulativos podendo levar a morte do animal.

Desta forma, o presente trabalho teve como objetivo avaliar os parâmetros de desempenho e características de carcaças de fêmeas suínas submetidas a diferentes níveis de inclusão do farelo de algodão, assim como a sua viabilidade econômica.

\section{Material e Métodos}

Foram utilizadas 24 fêmeas suínas de linhagem comercial, com idade média inicial de 103 dias e peso vivo médio de 48,20 $\pm 5,44 \mathrm{~kg}$ (fase de crescimento) e idade média de 131 dias e peso vivo médio de 87,62 $\pm 6,72 \mathrm{~kg}$ (fase de terminação), após aprovação no Comitê de Ética para uso de Animais da UFRPE sob o protocolo 23082.006830/2009B09.

Os leitões foram adquiridos com um peso médio de $33 \mathrm{~kg}$ e após a instalação, os animais passaram por adaptação pré-experimental de 28 dias. Neste período receberam uma ração inicial suplementada com antiparasitário e adaptada às instalações experimentais. O período experimental foi de 28 dias na fase de crescimento e 37 dias na fase de terminação, com duração total de 65 dias.

Os animais foram alojados em baias individuais com 3,00 $\mathrm{m}^{2}$, em galpão de alvenaria com altura de pédireito de 2,10 metros, cobertas com telha cerâmica. As baias continham bebedouro tipo chupeta e comedouros de alumínio circular com 22 centímetros de diâmetro e $15 \mathrm{~cm}$ de profundidade.

O delineamento experimental foi em blocos casualizados, com quatro tratamentos e seis repetições, com um suíno por unidade experimental, sendo o critério dos blocos estabelecido de acordo com o peso inicial dos animais.

As rações experimentais para as fases de crescimento e terminação foram: $0 \%$ de FA (ração a base de milho e farelo de soja); $10 \%$ de FA (inclusão de $10 \%$ de farelo de algodão); $20 \%$ de FA (inclusão de $20 \%$ de farelo de algodão); $30 \%$ (inclusão de $30 \%$ de farelo de algodão). As composições nutricionais das rações experimentais na fase de crescimento e terminação são apresentadas nas Tabelas 1 e 2, respectivamente. As rações foram isoenergética e isoproteicas para atender as exigências mínimas estabelecidas para fêmeas suínas em crescimento e terminação de alto potencial genético com desempenho médio, segundo Rostagno et al. (2005).

Tabela 1: Composição alimentar e valores analisados das rações experimentais para a fase de crescimento

\begin{tabular}{|c|c|c|c|c|}
\hline \multirow{2}{*}{ Ingredientes $\%$} & \multicolumn{4}{|c|}{ Nível de inclusão do farelo de algodão, \% } \\
\hline & 0 & 10 & 20 & 30 \\
\hline Milho & 53,430 & 54,985 & 56,541 & 58,096 \\
\hline Farelo de Soja 45 & 13,400 & 11,217 & 9,033 & 6,850 \\
\hline Farelo de Algodão & 0,000 & 10,000 & 20,000 & 30,000 \\
\hline Farelo de Trigo & 29,000 & 19,417 & 9,833 & 0,250 \\
\hline Óleo de Soja & 1,990 & 2,200 & 2,410 & 2,620 \\
\hline Fosfato Bicálcio & 0,000 & 0,143 & 0,287 & 0,430 \\
\hline Calcário & 1,204 & 1,100 & 0,997 & 0,893 \\
\hline Sal Comum & 0,380 & 0,375 & 0,370 & 0,365 \\
\hline L-Lisina HCL & 0,275 & 0,253 & 0,232 & 0,212 \\
\hline DL-Metionina & 0,013 & 0,012 & 0,012 & 0,011 \\
\hline Premix Vitamínico ${ }^{1}$ & 0,100 & 0,100 & 0,100 & 0,100 \\
\hline Premix Mineral $^{2}$ & 0,100 & 0,100 & 0,100 & 0,100 \\
\hline Cygro $^{3}$ & 0,050 & 0,050 & 0,050 & 0,050 \\
\hline L-Treonina & 0,060 & 0,048 & 0,035 & 0,023 \\
\hline Total & 100,000 & 100,000 & 100,000 & 100,000 \\
\hline \multicolumn{5}{|l|}{ Valores Analisados } \\
\hline Extrato Etéreo \% & 6,24 & 6,96 & 7,13 & 8,44 \\
\hline Proteína Bruta, \% & 15,71 & 15,38 & 15,40 & 15,66 \\
\hline Fibra em Detergente Neutro \% & 19,40 & 20,44 & 21,66 & 22,36 \\
\hline Matéria Seca \% & 87,85 & 87,46 & 87,80 & 87,88 \\
\hline \multicolumn{5}{|l|}{ Valores Calculados } \\
\hline Energia digestível, kcal/kg. & 3235 & 3235 & 3235 & 3235 \\
\hline Proteína bruta, $\%$ & 15,28 & 15,28 & 15,27 & 15,27 \\
\hline Cálcio, \% & 0,551 & 0,551 & 0,551 & 0,551 \\
\hline Fósforo disponível, \% & 0,283 & 0,282 & 0,282 & 0,282 \\
\hline Lisina, $\%$ & 0,894 & 0,894 & 0,894 & 0,894 \\
\hline Metionina + Cistina, \% & 0,544 & 0,569 & 0,595 & 0,620 \\
\hline Fibra bruta, \% & 4,451 & 6,334 & 8,217 & 10,100 \\
\hline Gordura, \% & 5,137 & 6,014 & 6,892 & 7,769 \\
\hline
\end{tabular}

${ }^{1}$ Quantidade por kg de ração: Vit. A- 8.000 .000 UI; Vit. D32.000.000 UI; Vit. E- 10.000 mg; Vit. K3- 2.000 mg; Vit. B1- 1.880 $\mathrm{mg}$; Vit. B2- $5.000 \mathrm{mg}$; Vit B6- $1.500 \mathrm{mg}$; Vit. B12- $10.000 \mathrm{mg}$; Niacina $24.800 \mathrm{mg}$; Ac. Pantotênico $7.000 \mathrm{mg}$; Biotina $80 \mathrm{mg}$; Ac. Fólico $500 \mathrm{mg}$; Selênio $250 \mathrm{mg}$; Antioxidante $100.000 \mathrm{mg}$; Veículo Q.S.P. 1000 g.

${ }^{2}$ Quantidade/kg de ração: Mn - 60,0 mg; Fe - 30,0 mg; Zn - 60,0 mg; $\mathrm{Cu}$ - 9,0 mg; I - 1,0 mg 
${ }^{3}$ Cada $100 \mathrm{~g}$ do produto contém: Amônio de Maduramicina $(1,0 \mathrm{~g})$, Álcool benzílico (4,0 g), Óleo de milho refinado (3,3 g), Gérmen de milho $(100,0 \mathrm{~g})$.

Tabela 2: Composição alimentar e valores analisados das rações experimentais para a fase de terminação

\begin{tabular}{|c|c|c|c|c|}
\hline \multirow[t]{2}{*}{ Ingredientes \% } & \multicolumn{3}{|c|}{ Nível de inclusão do farelo de algodão, \% } & \multirow[b]{2}{*}{30} \\
\hline & 0 & 10 & 20 & \\
\hline Milho & 57,002 & 57,314 & 57,626 & 57,938 \\
\hline Farelo de Soja 45 & 8,360 & 5,970 & 3,580 & 1,190 \\
\hline Farelo de Algodão & 0,000 & 10,000 & 20,000 & 30,000 \\
\hline Farelo de Trigo & 30,000 & 21,667 & 13,333 & 5,000 \\
\hline Óleo de Soja & 2,265 & 2,683 & 3,102 & 3,520 \\
\hline Fosfato Bicálcio & 0,000 & 0,115 & 0,230 & 0,345 \\
\hline Calcário & 1,215 & 1,127 & 1,040 & 0,952 \\
\hline Sal Comum & 0,355 & 0,350 & 0,346 & 0,341 \\
\hline L-Lisina $\mathrm{HCl}$ & 0,220 & 0,201 & 0,183 & 0,164 \\
\hline DL-Metionina & 0,000 & 0,000 & 0,000 & 0,000 \\
\hline Premix Vitamínico ${ }^{1}$ & 0,400 & 0,400 & 0,400 & 0,400 \\
\hline Premix Mineral $^{2}$ & 0,100 & 0,100 & 0,100 & 0,100 \\
\hline Cygro $^{3}$ & 0,050 & 0,050 & 0,050 & 0,050 \\
\hline L-Treonina & 0,033 & 0,022 & 0,011 & 0,000 \\
\hline Total & 100,000 & 100,000 & 100,000 & 100,000 \\
\hline \multicolumn{5}{|l|}{ Valores Analisados } \\
\hline Extrato Etéreo, \% & 5,96 & 6,15 & 7,5 & 9,3 \\
\hline Proteína Bruta, \% & 13,38 & 13,00 & 13,10 & 13,31 \\
\hline Fibra em Detergente Neutro, \%. & 18,24 & 20,00 & 23,13 & 24,94 \\
\hline Matéria Seca, \% & 88,10 & 87,76 & 86,77 & 89,06 \\
\hline \multicolumn{5}{|l|}{ Valores Calculados } \\
\hline Energia digestível, $\mathrm{kcal} / \mathrm{kg}$. & 3231 & 3231 & 3231 & 3231 \\
\hline Proteína bruta, \% & 13,37 & 13,37 & 13,37 & 13,37 \\
\hline Cálcio, \% & 0,546 & 0,546 & 0,546 & 0,546 \\
\hline Fósforo disponível, \% & 0,280 & 0,280 & 0,280 & 0,280 \\
\hline Lisina, \% & 0,728 & 0,728 & 0,728 & 0,728 \\
\hline Metionina + Cistina, \% & 0,485 & 0,512 & 0,538 & 0,565 \\
\hline Fibra bruta, \% & 4,336 & 6,308 & 8,279 & 10,250 \\
\hline Gordura, \% & 5,490 & 6,570 & 7,650 & 8,730 \\
\hline
\end{tabular}

${ }^{1}$ Quantidade por kg de ração: Vit. A-. 5.000.000 UI; Vit. D3800.000 UI; Vit. E- 8.000 mg; Vit. K3- 1.000 mg; Vit. B1- 500 mg; Vit. B2- $2.000 \mathrm{mg}$; Vit B6- $500 \mathrm{mg}$; Vit. B12- $10.000 \mathrm{mg}$; Niacina $8.000 \mathrm{mg}$; Ac. Pantotênico $6.000 \mathrm{mg}$; Selênio $250 \mathrm{mg}$; Colina 85 $\mathrm{mg}$; Antioxidante $100.000 \mathrm{mg}$; Veículo Q.S.P. $1000 \mathrm{~g}$.

${ }^{2}$ Quantidade/kg de ração: Mn - 60,0 mg; Fe - 30,0 mg; Zn - 60,0 mg; $\mathrm{Cu}$ - 9,0 mg; I - 1,0 mg

${ }^{3}$ Cada $100 \mathrm{~g}$ do produto contém: Amônio de Maduramicina $(1,0 \mathrm{~g})$, Álcool benzílico ( $4,0 \mathrm{~g})$, Óleo de milho refinado (3,3 g), Gérmen de milho $(100,0 \mathrm{~g})$.

As dietas e a água foram fornecidas à vontade durante todo período experimental, sendo as sobras e desperdícios das dietas pesados diariamente para avaliação do consumo diário.

Os animais foram pesados a cada 14 dias, ocasião em que foram tomadas, medidas do perímetro torácico e da espessura de toucinho com ultrassom modo A, tomadas por meio do aparelho RENCO LEAN - MEATER Digital Backfat indicator ${ }^{5}$, em três pontos da linha dorso lombar do animal, sempre a $6,5 \mathrm{~cm}$ abaixo da coluna vertebral na altura da quinta vértebra torácica, última vértebra torácica e da última vértebra lombar.

Ao final do experimento, por ocasião da pesagem, foram realizadas as medidas de ultrassom modo $\mathrm{B}$, as quais foram realizadas por meio do aparelho Aloka SSD 5006, utilizando-se um transdutor de 3,5 Mhz, tomadas no sítio P2, a $6,5 \mathrm{~cm}$ abaixo da linha média, na altura da última vértebra torácica, conforme metodologia descrita por Dutra Júnior et al. (2001)

No final do experimento foram escolhidos dois animais de acordo com o peso médio do tratamento num total de oito animais, para o abate. Os animais foram identificados e submetidos a jejum alimentar de 12 horas antes de embarcar para o abatedouro. Após o desembarque os animais ficaram

${ }^{5}$ Lean-Meater, USA.

${ }^{6}$ Aloka SSD-500 - Shangai Aloka Medical Equipment Ltda, China. nas baias de descanso e em jejum alimentar por mais 12 horas, e em jejum hídrico por seis horas. Antes do abate os animais foram submetidos à nova pesagem para a obtenção do peso ao abate após o jejum.

$\mathrm{O}$ abate envolveu as seguintes etapas: atordoamento por concussão cerebral, sangria com duração de três minutos com suspensão da carcaça, depilação com água quente, higienização, abertura da linha alba, evisceração, pesagem e separação das vísceras brancas e vermelhas, toalete e pesagem da carcaça quente (PCQ).

Em seguida as carcaças foram resfriadas em câmara fria com temperatura entre 2 e $4^{\circ} \mathrm{C}$ por 24 horas. Após 24 horas de resfriamento as carcaças foram serradas longitudinalmente, obtendo assim as meias carcaças, e em seguida pesadas para a obtenção do peso de carcaça fria (PCF). Foi calculada a porcentagem de perda de peso (PPP), com a relação entre PCQ e PAB e o rendimento de abate, relacionados ao $\mathrm{PCF}$ e $\mathrm{PCQ}$, assim como a porcentagem de perda por resfriamento $(\mathrm{PPR}=(\mathrm{PCQ}-\mathrm{PCF}) * 100 / \mathrm{PCQ})$.

O comprimento da carcaça (CC) foi tomado do bordo cranial da sínfise pubiana ao bordo crânio ventral do Atlas; a espessura de toucinho (ET) calculada por meio da média das medidas em três pontos da carcaça na primeira costela, última costela e última vértebra lombar, usando-se o valor médio obtido em três leituras consecutivas. A medida da área de olho de lombo (AOL) foi feita no mesmo local da espessura de toucinho na última costela. Neste ponto foi realizado um corte transversal, colocou-se um papel vegetal transparente e desenhou-se o contorno da área de olho de lombo, com a cobertura de gordura correspondente, incluindo a pele. Posteriormente foi calculada a área em escala milimétrica e foram feitos os cálculos para a área de carne e de gordura.

Os parâmetros avaliados foram: ganho de peso médio diário (GPD) $(\mathrm{kg})$, consumo de ração médio diário (CRD) $(\mathrm{kg})$, conversão alimentar $(\mathrm{CA})(\mathrm{kg} / \mathrm{kg})$; características de carcaças como peso e rendimento da carcaça fria (PCF, kg e RCF, \%), peso e rendimento da carcaça quente (PCQ, kg e RCQ, \%), área de olho de lombo medida pelo ultrassom (AOLUS) $\left(\mathrm{cm}^{2}\right)$, área de olho de lombo ao abate (AOLABATE) $\left(\mathrm{cm}^{2}\right)$, espessura de toucinho por ultrassom modo A (ETUS-A) (mm), espessura de toucinho por ultrassom modo B (ETUS-B) (mm), espessura de toucinho ao abate (ET-ABATE) (mm), relação Carne/Gordura $(\mathrm{kg} / \mathrm{kg})$, peso e rendimento dos cortes nobres (pernil, paleta, costela, carré e copa) (kg e \%) e parâmetros econômicos.

Os parâmetros econômicos avaliados foram: a receita bruta média (ganho de peso do suíno no período x preço pago por quilograma do suíno), custo médio da alimentação (consumo de ração total no período x preço da ração), margem bruta média (diferença entre a receita bruta média e o custo médio da alimentação) e a rentabilidade média (margem bruta média/ custo médio da alimentação) $\mathrm{x}$ 100 (BASTOS-LEITE et al., 2011).

Todos os parâmetros foram submetidos às análises de variância (ANOVA) e análises de regressão adotando modelos lineares e quadráticos ao nível de 5\% de probabilidade, utilizando o programa computacional SAEG (Universidade Federal de Viçosa, 2007). 


\section{Resultados e Discussão}

Os níveis de farelo de algodão não influenciaram significativamente $(\mathrm{P}>0,05)$ os dados de desempenho na fase de crescimento (Tabela 3). No entanto, o aumento dos níveis de farelo de algodão nas dietas dos animais reduziu linearmente $(\mathrm{P}<0,05)$ o ganho de peso diário (GPD) e consumo de ração diária (CRD) nas fases de terminação e total. O nível de $30 \%$ de inclusão de FA proporcionou menor consumo diário de ração e ganho de peso diário nas fases de terminação e total.

Tabela 3: Desempenho de fêmeas suínas alimentadas com diferentes níveis de inclusão do FA

\begin{tabular}{|c|c|c|c|c|c|c|c|}
\hline \multirow{2}{*}{ Variáveis } & \multicolumn{4}{|c|}{ Nível de inclusão \% } & \multirow[t]{2}{*}{$\mathrm{CV} \%$} & \multirow[t]{2}{*}{$P$} & \multirow[t]{2}{*}{$\mathrm{R}^{2}$} \\
\hline & 0 & 10 & 20 & 30 & & & \\
\hline \multicolumn{8}{|c|}{ Crescimento } \\
\hline GPD, kg & $1,02 \pm 0,07$ & $0,98 \pm 0,08$ & $1,00 \pm 0,04$ & $0,80 \pm 0,11$ & 8,26 & NS & - \\
\hline CRD, $\mathrm{kg}$ & $2,51 \pm 0,16$ & $2,43 \pm 0,23$ & $2,48 \pm 0,11$ & $2,34 \pm 0,17$ & 5,07 & NS & - \\
\hline $\mathrm{CA}$ & $2,45 \pm 0,22$ & $2,48 \pm 0,35$ & $2,50 \pm 0,07$ & $2,63 \pm 0,21$ & 10,28 & NS & - \\
\hline \multicolumn{8}{|c|}{ Terminação } \\
\hline GPD, $\mathrm{kg}^{(1)}$ & $0,95 \pm 0,06$ & $0,91 \pm 0,10$ & $0,90 \pm 0,07$ & $0,75 \pm 0,12$ & 10,57 & 0,009 & 0,82 \\
\hline CRD, $\mathrm{kg}^{(2)}$ & $3,22 \pm 0,10$ & $2,95 \pm 0,26$ & $3,00 \pm 0,24$ & $2,58 \pm 0,27$ & 8,26 & 0,003 & 0,81 \\
\hline $\mathrm{CA}$ & $3,37 \pm 0,20$ & $3,23 \pm 0,19$ & $3,33 \pm 0,16$ & $3,48 \pm 0,46$ & 8,84 & NS & 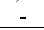 \\
\hline \multicolumn{8}{|c|}{ Total } \\
\hline GPD, $\mathrm{kg}^{(3)}$ & $0,64 \pm 0,02$ & $0,48 \pm 0,03$ & $0,48 \pm 0,04$ & $0,42 \pm 0,04$ & 6,45 & 0,001 & 0,96 \\
\hline CRD, $\mathrm{kg}^{(4)}$ & $2,91 \pm 0,09$ & $2,71 \pm 0,20$ & $2,78 \pm 0,18$ & $2,48 \pm 0,22$ & 6,28 & 0,004 & 0,78 \\
\hline 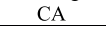 & $295 \pm 0.14$ & $2.88 \pm 0.19$ & $2,93 \pm 0,11$ & $3,05 \pm 0,23$ & 5,96 & & \\
\hline
\end{tabular}

CV: coeficiente de variação; $P$ : probabilidade; NS: não significativo; GPD: Ganho de peso diário; CRD: Consumo de ração diário; CA: conversão alimentar; (1) $\hat{Y}=0,973-0,006 X$; (2) $\hat{Y}=3,215-0,018 X$ (3) $\hat{Y}=0,644-0,032 X$; (4) $\hat{Y}=2,910-0,012 X$. *Valores se referem à média \pm desvio padrão entre as repetições.

O aumento do teor de fibra na ração dos animais pode ocasionar redução na ingestão voluntária de alimento devido ao rápido preenchimento gastrointestinal, o que pode ter comprometido a ingestão de energia pelos animais, uma vez que as dietas eram isoenergéticas (AGYEKUM; NYACHOTI, 2017). De acordo com a literatura (LINDBERG, 2014; ZANG et al., 2013), os suínos possuem uma maior capacidade de utilização da fibra na fase de terminação, devido ao maior desenvolvimento do trato gastrointestinal e maior número de bactérias intestinais. No entanto, na presente pesquisa, a idade das marrãs deve ser levada em consideração, pois o início da fase de terminação coincidiu com a entrada das marrãs na puberdade, fase a qual apresentaram o primeiro cio, havendo um estresse fisiológico aos animais, ocasionando a redução no consumo de ração e consequentemente no ganho de peso.

Os resultados de peso e rendimento de carcaça, espessura de toucinho por ultrassom (ETUS-A e ETUS-B) e relação carne/gordura não foram influenciados significativamente $(\mathrm{P}>0,05)$ pelos níveis de FA (Tabela 4). NO entanto, a variável espessura de toucinho ao abate (ETABATE) apresentou uma redução linear, sendo que a maior redução ocorreu no nível de $30 \%$ de inclusão de FA, com 9,3 $\mathrm{mm}$ versus os $14,0 \mathrm{~mm}$ do tratamento sem FA. A (este ponto e o A já estavam em vermelho, não os marquei) área de olho de lombo medida pelo ultrassom (AOL-US) apresentou um comportamento quadrático apresentando maior nível estimado de $11,12 \%$ e maior AOL-US de $47,48 \mathrm{~cm}^{2}$. A (este ponto e o A já estavam em vermelho, não os marquei) área de olho de lombo ao abate (AOL-ABATE) apresentou um comportamento quadrático apresentando maior nível estimado de $13,50 \%$ e maior AOL-US de $46,14 \mathrm{~cm}^{2}$.
Tabela 4: Parâmetros das características de carcaças de fêmeas suínas alimentadas com diferentes níveis de inclusão do FA

\begin{tabular}{ccccccc}
\hline & \multicolumn{9}{c}{ Nível de inclusão (\%) } & & \\
\cline { 2 - 5 } Variáveis & 0 & 10 & 20 & 30 & CV (\%) & P \\
\cline { 2 - 5 } PCQ (kg) & $81 \pm 1,34$ & $80,50 \pm 0,80$ & $82,62 \pm 3,40$ & $71,5 \pm 1,13$ & 3,26 & NS \\
RCQ $(\%)$ & $79,65 \pm 0,09$ & $78,41 \pm 0,42$ & $79,87 \pm 0,30$ & $75,77 \pm 1,96$ & 1,48 & NS \\
PCF $(\mathrm{kg})$ & $83,40 \pm 1,13$ & $83,45 \pm 1,07$ & $84,90 \pm 1,79$ & $73,7 \pm 1,27$ & 3,77 & NS \\
RCF $(\%)$ & $75,53 \pm 0,25$ & $74,37 \pm 0,16$ & $75,72 \pm 0,81$ & $71,37 \pm 1,70$ & 1,40 & NS \\
ETUS-A (mm) & $15,67 \pm 7,78$ & $12,83 \pm 3,54$ & $19,50 \pm 0,94$ & $15,08 \pm 1,53$ & 23,40 & NS \\
ETUS-B (mm) & $16,50 \pm 0,71$ & $13,60 \pm 1,84$ & $19,05 \pm 0,07$ & $13,60 \pm 2,55$ & 7,42 & NS \\
ET-ABATE (mm (m) & $14,00 \pm 0,03$ & $11,90 \pm 0,14$ & $13,80 \pm 1,13$ & $9,30 \pm 0,55$ & 4,80 & 0,01 \\
AOLUS $\left(\mathrm{cm}^{2}\right)^{(2)}$ & $42,35 \pm 1,63$ & $46,50 \pm 2,76$ & $46,05 \pm 0,42$ & $44,05 \pm 3,75$ & 3,33 & 0,01 \\
AOL - ABATE $\left(\mathrm{cm}^{2}\right)^{(3)}$ & $42,90 \pm 2,28$ & $46,77 \pm 7,76$ & $47,10 \pm 0,00$ & $36,45 \pm 3,19$ & 11,49 & 0,01 \\
Rel. Carne:Gordura & $0,47 \pm 0,02$ & $0,34 \pm 0,04$ & $0,48 \pm 0,07$ & $0,40 \pm 0,02$ & 10,00 & NS \\
\hline
\end{tabular}

CV: coeficiente de variação; P: probabilidade; NS: não significativo; PCQ: Peso de carcaça quente; RCQ: rendimento de carcaça quente; PCF: peso da carcaça fria; RCF: rendimento da carcaça fria; ETUS-A: espessura de toucinho ultrassom modo A; ETUS-B: espessura de toucinho ultrassom modo B; ET-ABATE: espessura de toucinho ao abate; AOLUS: área de olho de lombo ultrassom; AOL-ABATE: área de olho de lombo ao abate; (1) Linear: $\hat{Y}=15,3$ - 1,22X $\left(\mathrm{R}^{2}=0,72\right)$; (2) Quadrático: $\hat{\mathrm{Y}}=42,53+0,89 \mathrm{X}-0,04 \mathrm{X}^{2}$ $\left(\mathrm{R}^{2}=0,93\right)$; (3) Quadrático: $\hat{\mathrm{Y}}=42,50+0,54 \mathrm{X}-0,02 \mathrm{X}^{2}\left(\mathrm{R}^{2}=0,98\right)$. *Valores se referem à média \pm desvio padrão entre as repetições.

Segundo Gomes et al. (2008) a redução da espessura de toucinho representa atualmente uma das principais metas determinadas pela indústria frigorífica, em especial, quando a redução de tal parâmetro é acompanhada de elevação dos teores de carne magra e da área de olho de lombo na carcaça.

Segundo Kiefer (2014), a espessura de toucinho medida entre a última e a penúltima costela, apresenta alta correlação negativa com o percentual de carne magra, indicando que são características inversamente proporcionais, ou seja, o crescimento de uma inibe o desenvolvimento da outra característica, caracterizando mais uma vez a importância da diminuição da espessura de toucinho nos suínos terminados. Neste trabalho apesar da redução da ET e melhoria da AOL até o nível estimado de 13,5\% de inclusão do FA, não foi observada alteração na relação carne: gordura.

As diferenças nos valores absolutos da ET, para medidas tomadas na carcaça e medidas por ultrassom, podem ser explicadas pelo fato de que as medidas realizadas por ultrassom terem sido referentes à média de todos os animais das repetições e para as medidas tomadas nas carcaças, foram apenas dos animais abatidos, que foram escolhidos pelo peso médio de cada parcela.

Como se pode observar, não ocorreram variações nos rendimentos em quilogramas e em percentagem para todos os cortes realizados nas carcaças de fêmeas suínas alimentadas com rações contendo até $30 \%$ de inclusão de farelo de algodão (Tabela 5). Estes resultados podem estar relacionados à padronização do peso de abate dos animais, com exceção do tratamento com $30 \%$ de inclusão de FA que apresentaram pesos de carcaça inferiores aos demais tratamentos. Convém salientar também que os coeficientes de variação encontrados para os diferentes cortes foram baixos e estão de acordo com os resultados obtidos por Dutra Júnior et al. (2001). 
Tabela 5: Características de cortes comerciais em percentual (\%) e quilograma $(\mathrm{kg})$ em suínas fêmeas alimentadas com diferentes níveis de FA

\begin{tabular}{ccccccc}
\hline & \multicolumn{5}{c}{ Nível de inclusão (\%) } & \\
\cline { 2 - 5 } Variáveis & 0 & 10 & 20 & 30 & CV (\%) & P \\
\hline Pernil, kg & $23,2 \pm 0,28$ & $24 \pm 0,84$ & $23,4 \pm 0,42$ & $20,6 \pm 0,42$ & 4,72 & NS \\
Pernil, \% & $30,2 \pm 0,74$ & $31,43 \pm 2,22$ & $29,87 \pm 1,08$ & $30,58 \pm 1,26$ & 4,7 & NS \\
Paleta, $\mathrm{kg}$ & $19,2 \pm 0,28$ & $18,8 \pm 0,28$ & $19,6 \pm 0,28$ & $17,8 \pm 0,99$ & 5,86 & NS \\
Paleta, \% & $25 \pm 0,74$ & $24,62 \pm 0,74$ & $25,02 \pm 072$ & $26,43 \pm 2,94$ & 6,34 & NS \\
Costela, $\mathrm{kg}$ & $18,4 \pm 0,56$ & $18,8 \pm 0,84$ & $17,6 \pm 1,13$ & $17 \pm 0,42$ & 8,81 & NS \\
Costela, \% & $23,95 \pm 1,47$ & $24,62 \pm 2,22$ & $22,47 \pm 2,89$ & $25,24 \pm 1,26$ & 8,57 & NS \\
Carré, $\mathrm{kg}$ & $14 \pm 0,84$ & $12,75 \pm 0,31$ & $15,8 \pm 0,42$ & $11,8 \pm 0,14$ & 2,53 & NS \\
Carré, \% & $18,22 \pm 2,21$ & $16,7 \pm 0,83$ & $20,17 \pm 1,08$ & $17,52 \pm 0,42$ & 7,25 & NS \\
Copa, kg & $3,05 \pm 0,17$ & $2,43 \pm 0,41$ & $2,90 \pm 0,07$ & $2,53 \pm 0,01$ & 16,7 & NS \\
Copa, \% & $3,97 \pm 0,46$ & $3,18 \pm 1,08$ & $3,7 \pm 0,18$ & $3,76 \pm 0,04$ & 16,32 & NS \\
\hline
\end{tabular}

CV: coeficiente de variação; P: probabilidade; NS: não significativo. *Valores se referem à média \pm desvio padrão entre as repetições.

No presente trabalho, houve efeito das dietas sobre o peso e o rendimento do coração e fígado, além do rendimento dos rins, que aumentou linearmente $(\mathrm{P}<0,05)$ à medida que se elevou o nível de inclusão do farelo de algodão na ração (Tabela 6). Órgãos como fígado e rins possuem taxas metabólicas elevadas, sendo os seus respectivos aumentos de peso relacionados à maior inclusão de FA as dietas, podendo indicar uma adaptação dos animais às dietas.

Tabela 6: Média dos pesos e rendimentos dos órgãos internos das fêmeas suínas alimentadas com diferentes níveis de inclusão do FAAE

\begin{tabular}{|c|c|c|c|c|c|c|c|}
\hline \multirow[b]{2}{*}{ Variáveis } & & \multicolumn{4}{|c|}{ Nível de inclusão, \% } & \multirow{2}{*}{$\begin{array}{l}\text { CV } \\
(\%)\end{array}$} & \multirow{2}{*}{$P$} \\
\hline & & 0 & 10 & 20 & 30 & & \\
\hline \multirow[t]{2}{*}{ Coração } & $\mathrm{Kg}^{(1)}$ & $0,36 \pm 0,08$ & $0,38 \pm 0,09$ & $0,38 \pm 0,04$ & $0,66 \pm 0,01$ & 10,85 & 0,02 \\
\hline & & $0,37 \pm 0,08$ & $0,46 \pm 0,11$ & $0,48 \pm 0,01$ & $0,92 \pm 0,02$ & 12,80 & 0,01 \\
\hline \multirow{2}{*}{ Pulmão } & $\mathrm{Kg}$ & $0,51 \pm 0,07$ & $0,50 \pm 0,13$ & $0,49 \pm 0,06$ & $1,03 \pm 0,38$ & 34,09 & NS \\
\hline & $\%$ & $0,52 \pm 0,01$ & $0,63 \pm 0,16$ & $0,60 \pm 0,07$ & $1,40 \pm 0,53$ & 35,00 & NS \\
\hline \multirow[t]{2}{*}{ Rim } & $\mathrm{Kg}$ & $0,25 \pm 0,01$ & $0,25 \pm 0,07$ & $0,26 \pm 0,01$ & $0,24 \pm 0,01$ & 4,42 & NS \\
\hline & $\%^{(3)}$ & $0,24 \pm 0,01$ & $0,32 \pm 0,01$ & $0,31 \pm 0,01$ & $0,34 \pm 0,02$ & 4,53 & 0,01 \\
\hline \multirow[t]{2}{*}{$\overline{\text { Fígado }}$} & $\mathrm{Kg}^{(4)}$ & $1,37 \pm 0,01$ & $1,45 \pm 0,07$ & $1,44 \pm 0,01$ & $1,94 \pm 0,05$ & 1,70 & 0,01 \\
\hline & $\%^{(5)}$ & $1,38 \pm 0,01$ & $1,80 \pm 0,01$ & $1,74 \pm 0,01$ & $2,71 \pm 0,07$ & 2,02 & 0,01 \\
\hline \multirow[t]{2}{*}{ Estomago } & $\mathrm{Kg}$ & $0,92 \pm 0,21$ & $0,75 \pm 0,08$ & $0,77 \pm 0,07$ & $0,91 \pm 0,22$ & 20,30 & NS \\
\hline & $\%$ & $0,92 \pm 0,21$ & $0,93 \pm 0,10$ & $0,93 \pm 0,09$ & $1,20 \pm 0,32$ & 19,80 & NS \\
\hline \multirow[t]{2}{*}{ Intestino } & $\mathrm{Kg}$ & $4,97 \pm 0,36$ & $5,14 \pm 0,93$ & $4,45 \pm 0,87$ & $5,49 \pm 0,85$ & 10,94 & NS \\
\hline & $\%$ & $5,00 \pm 0,36$ & $6,38 \pm 1,16$ & $5,40 \pm 1,07$ & $7,60 \pm 1,20$ & 16,50 & NS \\
\hline \multirow[t]{2}{*}{ Gordura } & $\mathrm{Kg}$ & $0,15 \pm 0,35$ & $0,16 \pm 0,01$ & $0,15 \pm 0,01$ & $0,17 \pm 0,08$ & 31,30 & NS \\
\hline & $\%$ & $0,16 \pm 0,02$ & $0,20 \pm 0,01$ & $0,20 \pm 0,06$ & $0,25 \pm 0,01$ & 30,80 & NS \\
\hline
\end{tabular}

CV: coeficiente de variação; P: probabilidade; NS: não significativo; (1) $\hat{Y}=0,23+0,08 x\left(R^{2}=0,65\right) ;(2) \hat{Y}=0,14+0,16 x\left(R^{2}=0,76\right)$; (3) $\hat{Y}=0,23+0,02 x\left(R^{2}=0,74\right) ;(4) \hat{Y}=1,13+0,16 x\left(R^{2}=0,69\right) ;(5)$ $\hat{Y}=0,93+0,39 x\left(R^{2}=0,80\right)$. $*$ Valores se referem à média \pm desvio padrão entre as repetições.

O gossipol é um alcaloide polifenólico com pigmentação amarelada, facilmente encontrada em subprodutos do algodão, podendo ser tóxico a animais não ruminantes. O gossipol após absorvido pelo organismo será excretado $77 \%$ pelas fezes, sendo a bile a principal via para sua eliminação, $12 \%$ como gás carbônico e $3 \%$ pela urina, levando em torno de 78 horas para eliminá-lo do organismo, sendo o fígado, coração e rins, os tecidos que tendem a concentrar maiores teores de gossipol (ABOU-DONIA, 1976).

O aumento de peso do fígado, coração e rins pode estar associado a um elevado esforço desses órgãos para eliminar o gossipol livre na corrente sanguínea, visto que quanto maior o nível de FA, maior carga metabólica esses órgãos foram expostos.

A avaliação dos parâmetros econômicos (Tabela 7) demonstrou que houve um comportamento linear decrescente $(\mathrm{P}<0,05)$ para renda bruta média $(\mathrm{RBM})$, nas fases de crescimento, terminação e total, como também para o custo médio de alimentação (CMA) para a fase de terminação e total e para margem bruta média (MBM) na fase total à medida que se elevou os níveis de inclusão de farelo de algodão.

Tabela 7: Médias dos parâmetros de avaliação econômica para os períodos experimentais

\begin{tabular}{|c|c|c|c|c|c|c|}
\hline \multirow{2}{*}{ Variáveis } & \multicolumn{4}{|c|}{ Nível de inclusão, \% } & \multirow[b]{2}{*}{$\mathrm{CV} \%$} & \multirow[b]{2}{*}{$\mathrm{P}^{*}$} \\
\hline & 0 & 10 & 20 & 30 & & \\
\hline \multicolumn{7}{|c|}{ Crescimento } \\
\hline $\mathrm{RBM}^{(1)}$ & $107,62 \pm 7,77$ & $102,88 \pm 8,36$ & $104,94 \pm 3,70$ & $93,81 \pm 11,18$ & 8,01 & 0,045 \\
\hline CMA & $51,99 \pm 3,33$ & $50,30 \pm 4,72$ & $51,77 \pm 2,19$ & $48,80 \pm 3,47$ & 6,98 & NS \\
\hline MBM & $55,62 \pm 8,03$ & $52,58 \pm 9,98$ & $53,17 \pm 2,46$ & $45,01 \pm 8,57$ & 15,12 & NS \\
\hline RM & $107,56 \pm 17,92$ & $106,19 \pm 25,97$ & $102,83 \pm 5,48$ & $91,93 \pm 14,29$ & 17,16 & NS \\
\hline \multicolumn{7}{|c|}{ Terminação } \\
\hline $\mathrm{RBM}^{(2)}$ & $132,36 \pm 7,96$ & $126,06 \pm 13,26$ & $124,88 \pm 9,67$ & $104,00 \pm 16,24$ & 10,02 & 0,003 \\
\hline $\mathrm{CMA}^{(3)}$ & $88,58 \pm 2,87$ & $81,09 \pm 7,20$ & $83,28 \pm 6,64$ & $72,04 \pm 7,47$ & 7,78 & 0,001 \\
\hline MBM & $43,78 \pm 7,84$ & $44,97 \pm 8,17$ & $41,61 \pm 5,73$ & $31,97 \pm 12,82$ & 22,22 & NS \\
\hline RM & $49,50 \pm 9,21$ & $55,46 \pm 9,16$ & $50,14 \pm 7,13$ & $44,46 \pm 16,36$ & 22,12 & NS \\
\hline \multicolumn{7}{|c|}{ Total } \\
\hline $\mathrm{RBM}^{(4)}$ & $239,98 \pm 9,45$ & $228,95 \pm 11,91$ & $229,82 \pm 11,76$ & $197,81 \pm 23,17$ & 6,71 & 0,001 \\
\hline $\mathrm{CMA}^{(5)}$ & $140,80 \pm 4,28$ & $131,71 \pm 9,81$ & $135,47 \pm 8,53$ & $121,34 \pm 10,87$ & 6,60 & 0,007 \\
\hline $\mathrm{MBM}^{(6)}$ & $99,18 \pm 9,99$ & $97,24 \pm 11,21$ & $94,35 \pm 6,98$ & $76,47 \pm 16,14$ & 12,59 & 0,010 \\
\hline $\mathrm{RM}$ & $70,56 \pm 8,06$ & $74,38 \pm 11,97$ & $69,84 \pm 6,26$ & $63,01 \pm 11,51$ & 14,03 & NS \\
\hline
\end{tabular}

CV: coeficiente de variação; P: probabilidade; NS: não significativo; RBM: receita bruta média; CMA: custo médio da alimentação; MBM: margem bruta média; RM: rentabilidade média; (1) $\hat{Y}=$ 112,16 - 3,94X $\left(\mathrm{R}^{2}=0,72\right)$; (2) $\hat{\mathrm{Y}}=143,39-8,63 \mathrm{X}\left(\mathrm{R}^{2}=0,81\right)$; (3) $\hat{Y}=93,10-4,74 X\left(R^{2}=0,79\right) ;(4) \hat{Y}=255,55-12,56 X\left(R^{2}=0,79\right)$ (5) $\hat{Y}=145,99-5,46 X\left(R^{2}=0,73\right) ;(6) \hat{Y}=109,57-7,10 X\left(R^{2}=0,77\right)$. *Valores se referem à média \pm desvio padrão entre as repetições.

Os resultados também evidenciaram que a rentabilidade média para as fases de crescimento, terminação e total, não foram afetadas significativamente, uma vez que o custo da alimentação foi menor com o aumento da inclusão de FA.

O custo médio da alimentação (CMA) na fase de crescimento (Tabela 7) não foi afetado, uma vez que o consumo de ração (CR) nesta mesma fase (Tabela 3), também não foi modificado significativamente $(\mathrm{P}<0,05)$. $\mathrm{Na}$ fase de terminação e total, ocorreu uma diminuição linear no consumo de rações, onde este consumo de ração pode estar relacionado com a fase e condições corporais desses animais e consequentemente, gerando um baixo custo na alimentação.

\section{Conclusão}

É viável a inclusão de até 30\% do FA em dietas de fêmeas suína nas fases de crescimento, sem comprometer o parâmetro de desempenho, e de até $13,5 \%$ do FA para a fase de terminação sem interferir no desempenho, nas características de carcaça, peso dos órgãos e viabilidade econômica.

\section{Agradecimentos}

Os autores agradecem ao Instituto Nacional de Ciência e Tecnologia em Ciência Animal (INCT/CA CNPq).

\section{Referências}

ABOU-DONIA, M. B. Physiological effects and metabolism of gossypol. Residue Reviews, v. 61, p. 125160, 1976.

AGYEKUM, A. K.; NYACHOTI, C. M. Nutritional and metabolic consequences of feeding high-fiber diets to swine: A review. Engineering, v. 3, p.716-725, 2917. 
BARROS, L. V. et al. Replacement of soybean meal by cottonseed meal 38\% in multiple supplements for grazing beef heifers. Revista Brasileira de Zootecnia, v. 40, n. 4, p. 852-859, 2011.

BASTOS-LEITE, S. C. et al. Análise econômica da produção de leitões em período de creche submetidos a dietas à base de sorgo-soja, enriquecidas com caseína e lactose e isoladas. Informações Econômicas, v. 41, n. 8, p. 26-33, 2011.

BLEVINS, S. et al. Effects of silymarin on gossypol toxicosis in divergent lines of chickens. Poultry Science, v. 89, p. 1878-1886, 2010.

BORUAH, K. et al. Feed conversion efficiency (FCE) in relation to reproductive performance of sow. International Journal of Current Microbiology and Applied Sciences, v. 7, n. 1, p. 2915-2919, 2018.

DUTRA JR., W. M. et al. Estimativas de rendimentos de cortes comerciais e de tecidos de suínos em diferentes pesos de abate pela técnica de ultra-sonografia em tempo real.

Revista Brasileira de Zootecnia, v. 30, n. 4, p. 1243-1250, 2001.

GOMES, J. D. F. et al. Efeitos do incremento de fibra dietética sobre digestibilidade, desempenho e características de carcaça: II. Fêmeas suínas em pré-puberdade e puberdade. Semina: Ciências Agrárias, v. 28, n. 4, p. 727$738,2007$.

GOMES, J. D. F. et al. Desempenho e características de carcaça de suínos alimentados com dieta com feno de tifton (Cynodon Dactylon). Ciência Animal Brasileira, v. 9, n. 1, p. 59-67, 2008.

KIEFER, C. et al. Avaliação de carcaça de suínos imunocastrados pela técnica da ultrassonografia em tempo real. Revista Agrarian, v. 7, n. 23, p. 118-123, 2014.

LINDBERG, J. E. Fiber effects in nutrition and gut health in pigs. Journal of Animal Science and Biotechnology, v. 5, n. 15, p. 1-7, 2014.

MOREIRA, I. et al. Utilização do farelo de algodão, com ou sem a adição de ferro, na alimentação de leitões na fase inicial (15-30 kg). Revista Brasileira de Zootecnia, v. 35, n. 3, p. 1077-1084, 2006.

NEPOMUCENO, R. C. et al. Neutral detergent fibre in piglet diets: performance and gastrointestinal implications. Ciências e Agrotecnologia, v. 40, n. 2, p. 205-216, 2016.

PAIANO, D. et al. Farelos de algodão com diferentes níveis de proteína na alimentação de suínos na fase inicial: Digestibilidade e desempenho. Acta Scientiarum Animal Sciences, v. 28, n. 4, p. 415-422, 2006.

ROSTAGNO, H. S. et al. Tabelas brasileira para aves e suínos: composição de alimentos e exigências nutricionais. 2. ed. Viçosa, MG: UFV, 2005.

ROSTAGNO, H. S. et al. Tabelas brasileira para aves e suínos: composição de alimentos e exigências nutricionais. 4. ed. Viçosa, MG: UFV, 2017.

SAEG. Sistema de análises estatísticas e genéticas Versão 9.1. Universidade federal de Viçosa-UFV. Viçosa, MG, 2007.

THORNTON, P. K. Livestock production: recent trends, future prospects. Philosophical Transactions of the Royal Society of London. Series B, Biological Science, v. 265, n. 1554, p. 2853-2867, 2010.

ZANG, W. et al. The effects of dietary fiber level on nutriente digestibility in growing pigs Journal of Animal Science and Biotechnology, v. 4, n. 17, p. 1-7, 2013.

Recebido em: 29/11/2018 Aceito em: 23/06/2020 